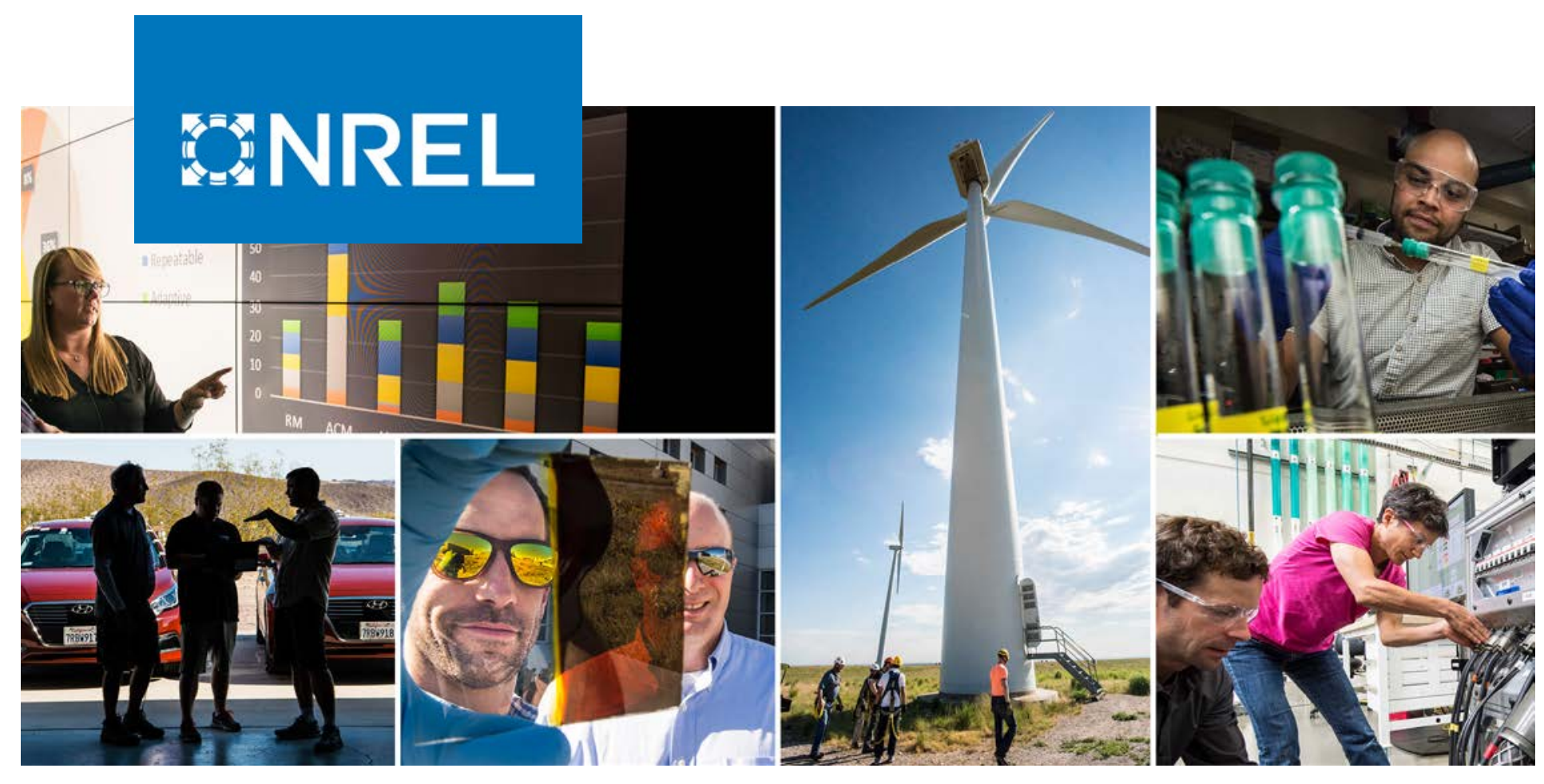

\title{
Slope-Aware Backtracking for Single-Axis Trackers
}

Kevin Anderson ${ }^{1}$ and Mark Mikofski ${ }^{2}$

1 National Renewable Energy Laboratory $2 D N V G L$

NREL is a national laboratory of the U.S. Department of Energy Office of Energy Efficiency \& Renewable Energy

Operated by the Alliance for Sustainable Energy, LLC

This report is available at no cost from the National Renewable Energy Laboratory (NREL) at www.nrel.gov/publications.
Technical Report

NREL/TP-5K00-76626

July 2020 


\section{GNREL}

\section{Slope-Aware Backtracking for Single-Axis Trackers}

Kevin Anderson ${ }^{1}$ and Mark Mikofski ${ }^{2}$

1 National Renewable Energy Laboratory

$2 D N V G L$

\section{Suggested Citation}

Anderson, Kevin and Mark Mikofski. 2020. Slope-Aware Backtracking for Single-Axis Trackers.

Golden, CO: National Renewable Energy Laboratory. NREL/TP-5K00-76626.

https://www.nrel.gov/docs/fy20osti/76626.pdf.

NREL is a national laboratory of the U.S. Department of Energy Office of Energy Efficiency \& Renewable Energy Operated by the Alliance for Sustainable Energy, LLC

This report is available at no cost from the National Renewable Energy Laboratory (NREL) at www.nrel.gov/publications.

Contract No. DE-AC36-08GO28308
Technical Report NREL/TP-5K00-76626 July 2020

National Renewable Energy Laboratory 15013 Denver West Parkway Golden, CO 80401 303-275-3000 • www.nrel.gov 


\section{NOTICE}

This work was authored in part by Alliance for Sustainable Energy, LLC, the manager and operator of the National Renewable Energy Laboratory for the U.S. Department of Energy (DOE) under Contract No. DE-AC36-08GO28308. Funding provided by the U.S. Department of Energy's Office of Energy Efficiency and Renewable Energy (EERE) under Solar Energy Technologies Office (SETO) Agreement Numbers 34348. The views expressed in the article do not necessarily represent the views of the DOE or the U.S. Government. The U.S. Government retains and the publisher, by accepting the article for publication, acknowledges that the U.S. Government retains a nonexclusive, paid-up, irrevocable, worldwide license to publish or reproduce the published form of this work, or allow others to do so, for U.S. Government purposes.

Neither DNV GL nor any group company (the "Group") assumes any responsibility whether in contract, tort (including without limitation negligence), or otherwise and no company in the Group including DNV GL shall be liable for any loss or damage whatsoever. This document is issued on a no reliance basis and nothing in this document guarantees any particular energy resource or system output.

This report is available at no cost from the National Renewable Energy Laboratory (NREL) at www.nrel.gov/publications.

U.S. Department of Energy (DOE) reports produced after 1991 and a growing number of pre-1991 documents are available free via www.OSTI.gov. 


\section{Acknowledgments}

The authors would like to thank Cliff Hansen and Matt Muller for significantly improving this report with their review and helpful suggestions on the technical content and presentation. 


\section{Nomenclature}

$\beta_{a} \quad$ Axis tilt, angle of inclination from horizontal of the tracker axes, $0^{\circ}$ to $+90^{\circ}$.

$\beta_{c} \quad$ Cross-axis slope angle, angle of inclination from horizontal of the plane containing the tracker axes, in the cross-axis direction, $-90^{\circ}$ to $+90^{\circ}$.

$\beta_{s} \quad$ Solar elevation angle, angle of sun above the horizontal, $0^{\circ}$ to $+90^{\circ}$.

$\beta_{g} \quad$ Grade slope angle, angle between slope plane and horizontal plane, $0^{\circ}$ to $+90^{\circ}$.

$\gamma_{a} \quad$ Axis azimuth, angle clockwise from north of the horizontal projection of the tracker axis, $0^{\circ}$ to $+360^{\circ}$.

$\gamma_{g} \quad$ Grade azimuth, angle clockwise from north of the horizontal projection of falling slope, $0^{\circ}$ to $+360^{\circ}$.

$\gamma_{s} \quad$ Solar azimuth, angle of sun clockwise from north, $0^{\circ}$ to $+360^{\circ}$.

$\theta_{B} \quad$ Backtracking rotation, deviation from flat in the rotation plane, $-180^{\circ}$ to $+180^{\circ}$.

$\theta_{c} \quad$ Backtracking correction angle, difference between true-tracking and backtracking angles, $-180^{\circ}$ to $+180^{\circ}$.

$\theta_{T} \quad$ True-tracking rotation, deviation from flat in the rotation plane, $-180^{\circ}$ to $+180^{\circ}$.

$f_{s} \quad$ Shaded fraction, the fraction of a row that is shaded by the adjacent row, 0 to 1 .

GCR Ground coverage ratio, the ratio of tracker collector width $\ell$ to row pitch $p, 0$ to 1 .

$h \quad$ Row offset, the vertical on-center z-distance between adjacent tracker axes.

$\ell \quad$ Collector width, the cross-axis distance spanned by the tracker's solar modules.

$p \quad$ Row pitch, the horizontal on-center distance between adjacent tracker axes. 


\section{Table of Contents}

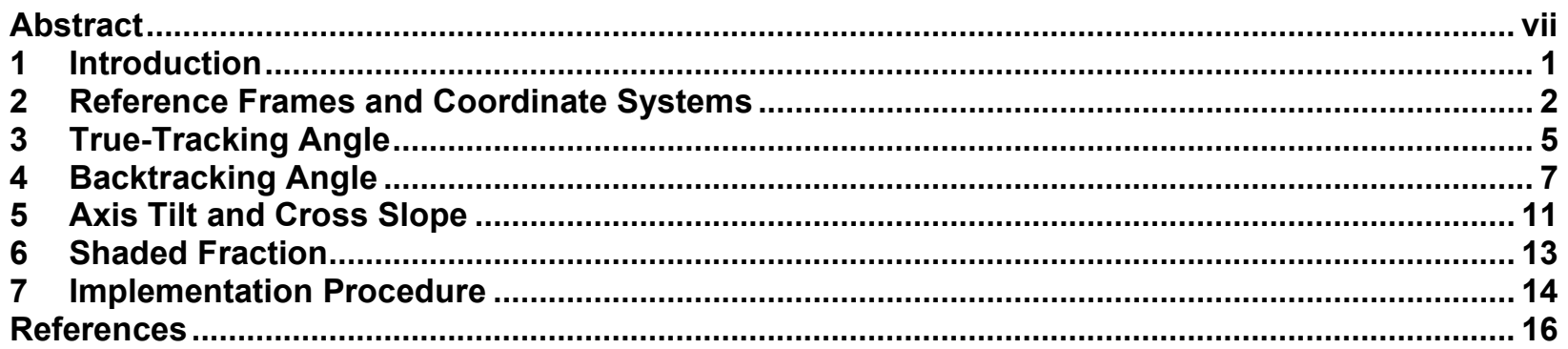

\section{List of Figures}

Figure 1: Comparison between global coordinate axes (blue) and tracker coordinates axes (red). The global coordinates $x, y, z$ are defined by east, north, and up. The tracker coordinates $t x, t y, t z$ are defined by rotating by the tracker axis azimuth $\gamma a$ and tilt $\beta a$.

Figure 2: Solar position angles and the corresponding Cartesian coordinates. The global Cartesian coordinates are found by treating the solar position angles as spherical coordinates. In this case, the corresponding Cartesian $x$ and $y$ coordinates are positive and negative, respectively (sun in eastern and southern sky).

Figure 3: Tracker rotation $\theta$ relative to the tracker coordinate system. The rotation is a right-handed rotation around $t y$ equal to the angle from $t z$ to the module normal (shown in black). Because the rotation is right-handed, reversing the direction of $t y$ (for example from roughly south to north) determines which direction (west or east respectively) is considered a positive rotation. This example shows a positive rotation to the west assuming $t y$ is roughly south. 4

Figure 4: Cross-axis slope angle $\beta c$ relative to the tracker coordinate system. The rotation is a right-handed rotation around $t y$. Because the rotation is righthanded, reversing the direction of $t y$ (from roughly south to north) determines which direction of slope (west or east respectively) is considered a positive cross-axis slope angle. This example shows a positive rotation to the west assuming ty is roughly south.

Figure 5. Projecting solar position onto the tracker rotation plane. AB shows a tracker axis with its tracker rotation plane defined by the $t x$ and $t z$ axes. The projected solar coordinates $\left(s x^{\prime}, s z^{\prime}\right)$ are found by rotating the solar Cartesian coordinates ( $s x, s y, s z)$ into the tracker coordinate system. Note that in this case, projected solar coordinate $s x^{\prime}$ is negative, meaning $\theta T<0$.

Figure 6. Cross-section of adjacent single-axis tracker rows with an offset $h$. The tracker axes point into the page and are not visible in this 2D diagram. The slopeaware backtracking position is shown in position $D$, with the true-tracking position as $F$ for comparison. Point $E$ shows the midpoint of the line segment (not shown) connecting the tracker axes, i.e. the segment between point $A$ and the corresponding point on the left tracker.

Figure 7. Cross-section of two adjacent tracker rows. The tracker axes point into the page and are not visible in this 2D diagram. The two optimal positions P1 and P2 eliminate row to row shading. P1 and P2 expose the same cross section to 
beam irradiance and are symmetrical around the true-tracking position, shown as a dotted line perpendicular to the solar vector.....

Figure 8. Geometry of a single-axis tracking axis $A B$ on a slope which is non-parallel to that axis. The vector $v$ is perpendicular to $A B$ and parallel to the slope plane, representing the cross-axis slope vector. Note that the $(x, y, z)$ axes here are arbitrarily chosen to align with the slope.

Figure 9. A cross-section of two adjacent tracker rows on a cross-axis slope. The tracker axes point into the page and are not visible in this 2D diagram. The tracker on the right is offset by $h$.

Figure 10: Example true-tracking and backtracking curves for various array orientations. 


\section{Abstract}

Closed-form equations of the true-tracking angle, backtracking angle, shaded fraction, and orientation angles of single-axis solar trackers installed on arbitrarily oriented slopes are derived. These slope-aware adjustments are necessary to successfully prevent row to row shading in arrays with nonzero cross-axis slope. A tracker rotation modeling procedure comprising these equations is provided. 


\section{Introduction}

Solar trackers optimize collector orientation and PV yield by rotating collectors to track the sun's movement across the sky. As described by Marion and Dobos (2013), the main objective of a tracker control algorithm is to maximize collector exposure to direct beam irradiance from the sun by minimizing the beam irradiance angle of incidence. However, this behavior will cause adjacent rows to partially shade each other across part of the module when the sun is low in the sky. For certain module types where such a shadow is distributed across all cells equally (for instance, thin film modules), this is an acceptable loss linearly proportional to fraction of shade. However, conventional modules with crystalline silicon cells are usually arranged in such a way that the shadow falls entirely on a few cells, creating significant production loss due to electrical mismatch. In that case, the array should operate at increased angle of incidence when the sun is low in the sky to prevent row-to-row shading. This behavior is called "backtracking" because it is accomplished by the tracker rotating backwards from the "ideal" rotation so that the row's shadow is shortened and misses the row behind it. The optimal backtracking rotation sacrifices as little beam irradiance as possible and is calculated using the spacing geometry between rows and the instantaneous solar position so that each row's shadow extends to, but not onto, the row behind. However, the backtracking geometry used in many commercial single-axis tracker systems is equivalent to the one described by Lorenzo, Narvarte, and Muñoz (2011), which assumes tracker axes are contained within a horizontal plane, i.e.: there is no vertical offset between rows. This is often a valid assumption for real-world PV systems, but for systems installed on even mild cross-slopes, the harsh nonlinearity between shading and power loss motivates a more general backtracking method that considers both horizontal and vertical row offsets. Slope-aware backtracking has been explored briefly by Nascimento et al. (2015) and Schneider (2012), but here we present a more comprehensive mathematical treatment.

Section 2 describes the various reference frames and coordinate systems used in later sections. Section 3 provides a derivation of the ideal rotation angle that minimizes beam irradiance angle of incidence for an arbitrarily oriented single-axis tracker axis. Section 4 derives a general single-axis backtracking formula that prevents row to row shading in arrays on sloped terrain by accounting for the component of the array's slope that is perpendicular to the tracker axes. Section 5 provides formulas for the required slope-adjusted orientation inputs (tracker axis inclination angle and cross-axis slope angle) from base array and slope orientation. Section 6 provides a formula of calculating the shaded fraction of a row based on single-axis tracker rotation angle, array geometry, and slope geometry. Finally, Section 7 outlines a step-by-step procedure to combine these equations to model single-axis tracker rotations.

The tracker rotation and auxiliary equations presented here have applications in PV performance simulations, O\&M monitoring, and the control software used in field single-axis tracker units. All equations presented here are closed-form and the final expressions are not substantially more complex than the standard backtracking equations, so it should be straightforward to incorporate them into modeling and tracker controller software. 


\section{Reference Frames and Coordinate Systems}

Before deriving tracking strategy equations, it is worthwhile to take a moment to explain the relevant reference frames and coordinate systems. Careless treatment of these details could end up with tracker systems following the sun the wrong way across the sky or backtracking according to a slope in the wrong direction. We draw attention to details like the distinction between north-azimuth and south-azimuth tracker axes $\left(\gamma_{a}=0^{\circ}\right.$ and $180^{\circ}$, respectively) and positive and negative tracker rotations. The reader should be familiar with the right-hand rule to determine the sign of angles and how coordinate axes relate to each other.

The first reference frame is the global reference frame of the location of the PV system in question, described with a right-handed Cartesian coordinate system with $x$ axis pointing east, $y$ axis pointing north, and $z$ axis pointing up. It is indifferent to the specifics of a PV system and acts as the fixed environmental frame that the local tracker reference frame is defined in relation to. The various azimuth angles (axis azimuth $\gamma_{a}$, solar azimuth $\gamma_{s}$, and grade azimuth $\gamma_{g}$ ) are defined as angles in this global coordinate system. For consistency with existing industry convention, azimuth is defined as a clockwise angle around the global $z$ axis, e.g. north is $0^{\circ}$, east is $90^{\circ}$, south is $180^{\circ}$, and west is $270^{\circ}$. Note that these are left-handed angles with respect to the global $z$ axis and need to be handled as such when used mathematically.

To describe rotations of the tracker plane, we define a second reference frame called the tracker reference frame. This frame is specific to the PV system and is defined by rotating the global frame by the system's axis azimuth $\gamma_{a}$ and axis tilt $\beta_{a}$. The tracker reference frame is described by the right-handed Cartesian coordinate system with coordinate axes $\overrightarrow{t_{x}}, \overrightarrow{t_{y}}, \overrightarrow{t_{z}}$, where $\overrightarrow{t_{x}}$ is horizontal and perpendicular to the tracker axis, $\overrightarrow{t_{y}}$ is parallel to the tracker axis and points in the direction of the tracker azimuth $\gamma_{a}$, and $\overrightarrow{t_{z}}$ is the cross product of $\overrightarrow{t_{x}}$ and $\overrightarrow{t_{y}}$, so it points roughly upward with $\beta_{a}$ as the angle between $\overrightarrow{t_{z}}$ and vertical $(z)$ in the global reference frame. Like the azimuth angles, the axis tilt is a left-handed angle around $\overrightarrow{t_{x}}$. Figure 1 shows the relationship between the tracker and global reference frames.
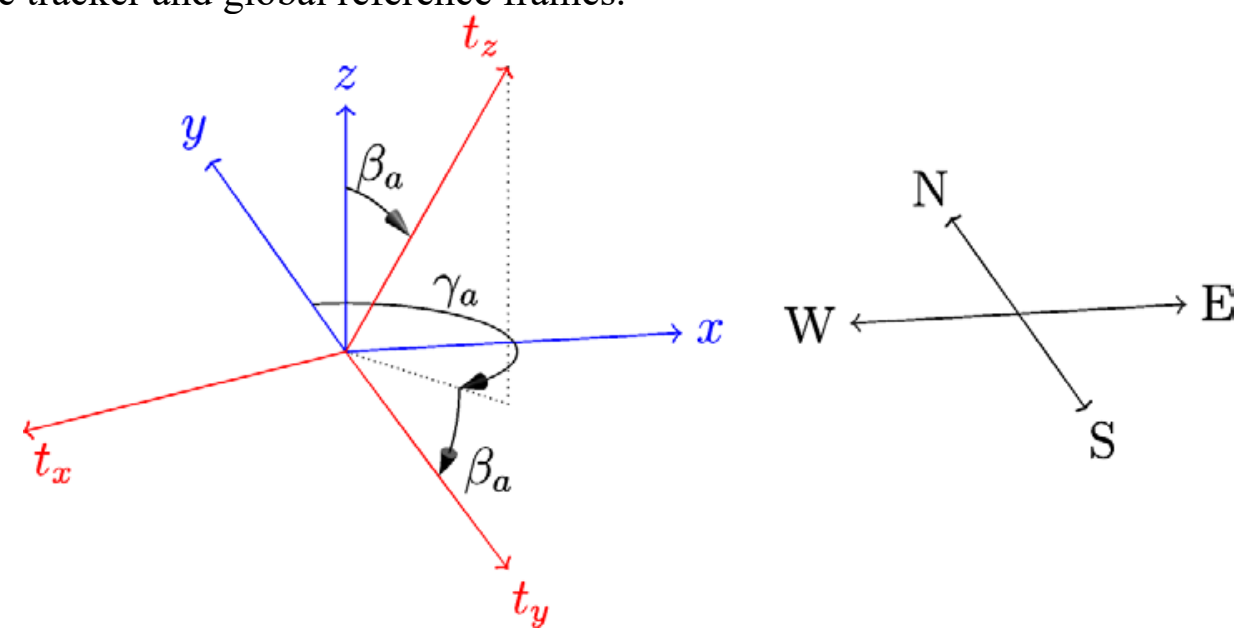

Figure 1: Comparison between global coordinate axes (blue) and tracker coordinates axes (red). The global coordinates $(x, y, z)$ are defined by east, north, and up. The tracker coordinates $\left(t_{x}, t_{y}, t_{z}\right)$ are defined by rotating by the tracker axis azimuth $\gamma_{a}$ and tilt $\beta_{a}$. 
Working in the tracker reference frame is convenient because it allows the system to be treated as if it was a simple north-south horizontal axis system. However, it does require external coordinates like solar position to be transformed into the tracker reference system. Because the common solar position calculation methods predict solar position in terms of its angular coordinates in the two-dimensional sky dome, we also consider a spherical coordinate system in the global reference frame. Figure 2 shows how these spherical coordinates relate to the global Cartesian coordinates.

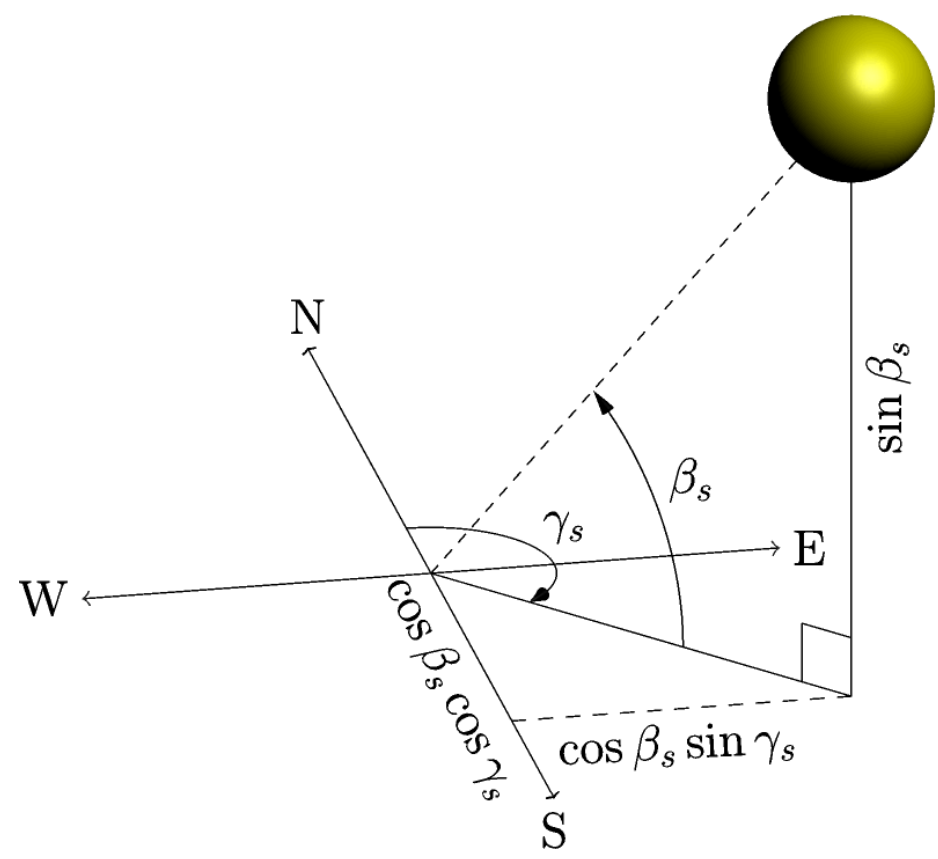

Figure 2: Solar position angles and the corresponding Cartesian coordinates. The global Cartesian coordinates are found by treating the solar position angles as spherical coordinates. In this case, the corresponding Cartesian $x$ and $y$ coordinates are positive and negative, respectively (sun in eastern and southern sky).

The sun's position is identified here with the azimuth and elevation angles $\gamma_{s}$ and $\beta_{s}$, noting that the elevation angle is the angular complement to the more common zenith angle.

Note that the system's physical placement and orientation are described equivalently by two axis azimuth angles separated by $180^{\circ}$. However, the choice of $\gamma_{a}$ determines the direction of the $\overrightarrow{t_{y}}$ axis and therefore also determines the sign convention for rotation angles. By the right-hand rule, a rotation around an axis is positive when it is counterclockwise around the positive axis. For example, consider a tracker array with $\gamma_{a}=180^{\circ}\left(\overrightarrow{t_{y}}\right.$ pointing south $)$ which places $\overrightarrow{t_{x}}$ pointed west. To orient the modules to the east in the morning, the rotation of the tracker around the $\overrightarrow{t_{y}}$ axis is negative, and so $\theta_{T}<0$. Similarly, $\theta_{T}>0$ in the afternoon to orient the modules to face west. For an array with the same geometry but $\gamma_{a}=0^{\circ}$, the reverse would be true: positive rotation in the morning and negative in the afternoon. For a right-handed rotation to be consistent with the industry convention of considering morning rotations negative and afternoon rotations positive, a north-south aligned system must have $\gamma_{a}=180^{\circ}$. Figure 3 shows this rotation in the context of the tracker coordinate system. 


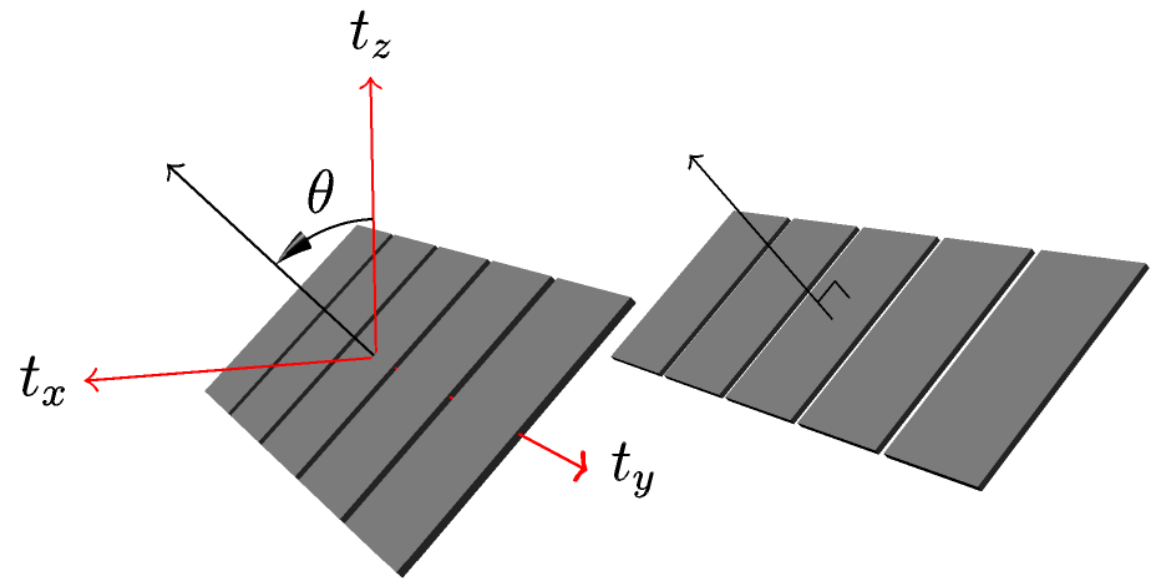

Figure 3: Tracker rotation $\theta$ relative to the tracker coordinate system. The rotation is a righthanded rotation around $\overrightarrow{t_{y}}$ equal to the angle from $\overrightarrow{t_{z}}$ to the module normal (shown in black). Because the rotation is right-handed, reversing the direction of $\overrightarrow{t_{y}}$ (for example from roughly south to north) determines which direction (west or east respectively) is considered a positive rotation.

This example shows a positive rotation to the west assuming $\overrightarrow{t_{y}}$ is roughly south.

Similarly, the orientation of $\overrightarrow{t_{y}}$ determines the sign of the cross-axis slope angle $\beta_{c}$. By defining $\beta_{c}$ as a right-handed rotation around $\overrightarrow{t_{y}}$ and using the convention determined above for tracker rotation angles, the right-hand rule determines west-facing slopes to have positive cross-axis slope for $\gamma_{a}=180^{\circ}$. This is shown in Figure 4.

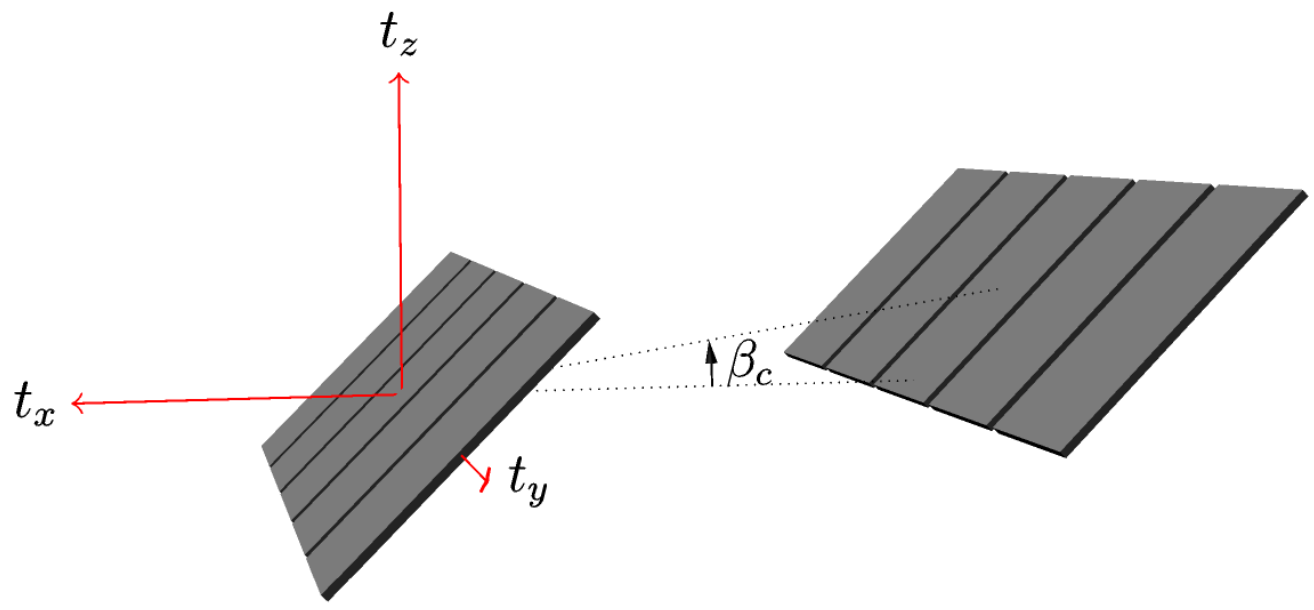

Figure 4: Cross-axis slope angle $\beta_{c}$ relative to the tracker coordinate system. The rotation is a right-handed rotation around $\overrightarrow{t_{y}}$. Because the rotation is right-handed, reversing the direction of $\overrightarrow{t_{y}}$ (from roughly south to north) determines which direction of slope (west or east respectively) is considered a positive cross-axis slope angle. This example shows a positive rotation to the west assuming $\overrightarrow{t_{y}}$ is roughly south. 


\section{True-Tracking Angle}

As described by Marion and Dobos (2013), the beam component of collected irradiance is maximized by minimizing its angle of incidence with the module normal. Because single-axis trackers cannot face directly towards the sun except in unusual circumstances, this minimization is instead achieved by matching tracker rotation to the projection of the sun's position onto the tracking plane of rotation, i.e.: the plane swept by the panel normal over the tracker's range of motion. This projection is easier to calculate if the solar position, which is usually reported in spherical coordinates, is expressed in Cartesian coordinates instead. As described in Figure 2, the solar azimuth $\gamma_{s}$ and elevation $\beta_{s}$ are converted from spherical coordinates to Cartesian coordinates with:

$$
\left[\begin{array}{l}
s_{x} \\
s_{y} \\
s_{z}
\end{array}\right]=\left[\begin{array}{c}
\cos \beta_{s} \sin \gamma_{s} \\
\cos \beta_{s} \cos \gamma_{s} \\
\sin \beta_{s}
\end{array}\right] .
$$

These Cartesian coordinates are used to calculate the solar projection onto the plane perpendicular to the tracker axis. Figure 5 shows a tracker axis $A B$ with tilt $\beta_{a}$ and azimuth $\gamma_{a}$. The perpendicular vectors $\overrightarrow{t_{x}}$ and $\overrightarrow{t_{z}}$ together define the tracking plane of rotation. The projected solar coordinates $\left(s_{x}^{\prime}, s_{z}^{\prime}\right)$ on the tracking plane of rotation determine $\theta_{T}$, the true-tracking rotation angle.

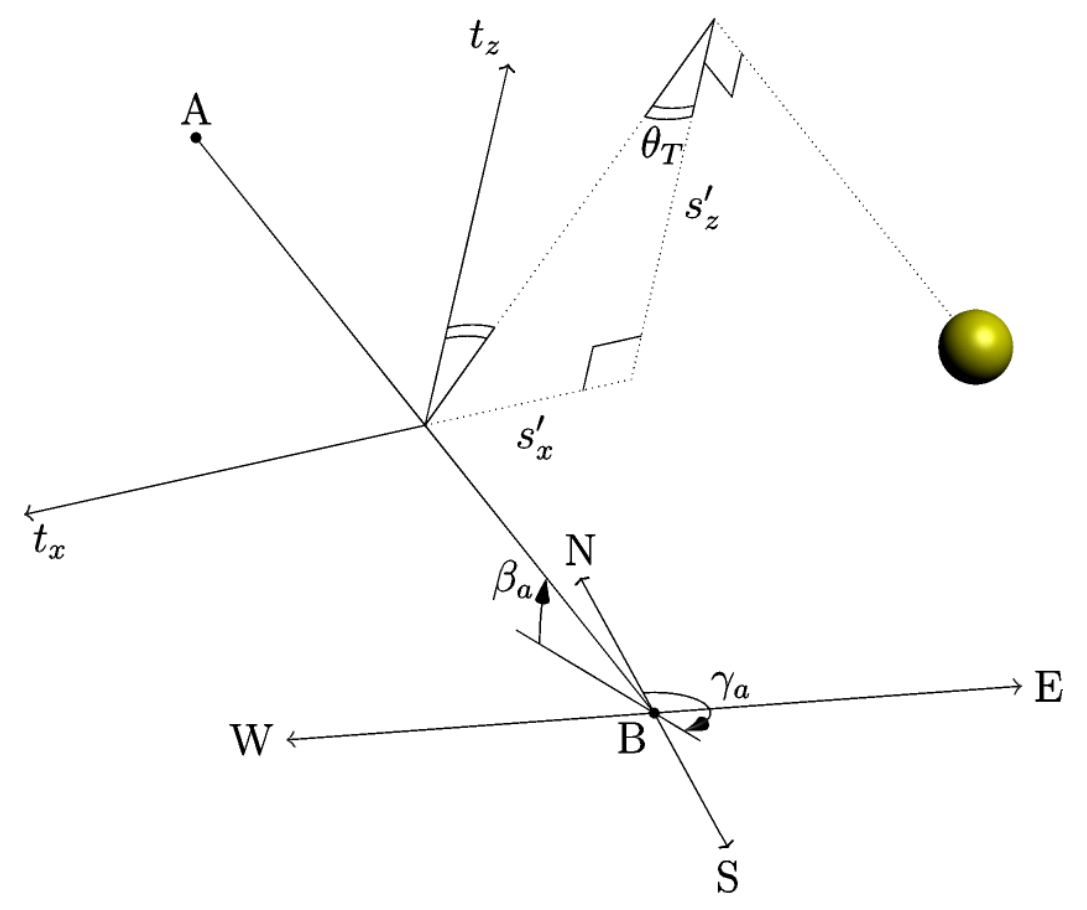

Figure 5. Projecting solar position onto the tracker rotation plane. AB shows a tracker axis with its tracker rotation plane defined by the $\overrightarrow{t_{x}}$ and $\overrightarrow{t_{z}}$ axes. The projected solar coordinates $\left(s_{x}^{\prime}, s_{z}^{\prime}\right)$ are found by rotating the solar Cartesian coordinates $\left(s_{x}, s_{y}, s_{z}\right)$ into the tracker coordinate system. Note that in this case, projected solar coordinate $s_{x}^{\prime}$ is negative, meaning $\theta_{T}<0$. 
Rotation matrices enable transformation from the global coordinates $(\vec{s})$ to the tracker coordinates $\left(\overrightarrow{s^{\prime}}\right)$ by first rotating around the $z$ axis by the axis azimuth and then around the $x$ axis by the axis tilt:

$$
\overrightarrow{s^{\prime}}=\mathbf{R}_{\mathbf{x}} \mathbf{R}_{\mathbf{z}} \vec{s}
$$

where:

$$
\mathbf{R}_{\mathbf{x}}=\left[\begin{array}{ccc}
1 & 0 & 0 \\
0 & \cos \beta_{a} & -\sin \beta_{a} \\
0 & \sin \beta_{a} & \cos \beta_{a}
\end{array}\right] \quad \text { and } \quad \mathbf{R}_{\mathbf{z}}=\left[\begin{array}{ccc}
\cos \gamma_{a} & -\sin \gamma_{a} & 0 \\
\sin \gamma_{a} & \cos \gamma_{a} & 0 \\
0 & 0 & 1
\end{array}\right]
$$

Note $\mathbf{R}_{\mathbf{x}}$ and $\mathbf{R}_{\mathbf{z}}$ are counterclockwise rotations around the global $x$ and $z$ axes, despite angles $\beta_{a}$ and $\gamma_{a}$ being clockwise angles. This is because the angles $\beta_{a}$ and $\gamma_{a}$ define the basis transformation from global to tracker coordinates, so their negative is used for vector transformations from global to tracker coordinates. This sign change cancels the sign change from converting the left-handed angles to right-handed angles. For ease of implementation, Equation 2 expands to:

$$
\left[\begin{array}{c}
s_{x}^{\prime} \\
s_{y}^{\prime} \\
s_{z}^{\prime}
\end{array}\right]=\left[\begin{array}{c}
s_{x} \cos \gamma_{a}-s_{y} \sin \gamma_{a} \\
s_{x} \sin \gamma_{a} \cos \beta_{a}+s_{y} \cos \beta_{a} \cos \gamma_{a}-s_{z} \sin \beta_{a} \\
s_{x} \sin \gamma_{a} \sin \beta_{a}+s_{y} \sin \beta_{a} \cos \gamma_{a}+s_{z} \cos \beta_{a}
\end{array}\right]
$$

Note, in Lorenzo, Narvarte, and Muñoz (2011), $s_{y}^{\prime}$ is missing $\sin \gamma_{a}$ from the first term, but it doesn't change their derivation because $s_{y}^{\prime}$ is never used. Because the in-plane components $s_{x}^{\prime}$ and $s_{z}^{\prime}$ define the projection of solar position onto the tracking rotation plane, the tracker rotation $\theta_{T}$ that faces the collector surface at the projected position is given by:

$$
\theta_{T}=\operatorname{atan} 2\left(s_{x}^{\prime}, s_{z}^{\prime}\right)
$$

where $\operatorname{atan} 2\left(s_{x}^{\prime}, s_{z}^{\prime}\right)$ is preferred over the more common $\tan ^{-1}\left(s_{x}^{\prime} / s_{z}^{\prime}\right)$ because it has an extended range of $\left(-180^{\circ}, 180^{\circ}\right.$, allowing $\theta_{T}$ to progress beyond $\pm 90^{\circ}$ when the sun is low in the sky and crosses "underneath" a tilted array. This tracker rotation is called the "true-tracking" angle. Note that Lorenzo et al. refer to the true-tracking angle as $\omega_{I D}$. It minimizes the angle of incidence between the collector surface and the sun's direct irradiance, thereby maximizing the capture of direct irradiance. 


\section{Backtracking Angle}

A system backtracks to avoid row to row shading while deviating as little as possible from the true-tracking angle in order to still optimize capture of direct irradiance. This is achieved by orienting the row such that the shadow cast from the top edge of one row is exactly tangent to the bottom edge of the row behind it. Equivalently, when the solar vector is projected onto the tracker rotation plane, it passes through a point on the upper edge of one row to a point on the lower edge of the previous row.

This derivation of backtracking angle for trackers on a cross-axis slope extends the derivation provided by Lorenzo, Narvarte, and Muñoz (2011) for trackers on a horizontal plane. Figure 6 shows a side view of two adjacent tracker rows on a cross-axis slope. The rows have collector width $l$ and are separated by a horizontal pitch $p$ and z-offset $h$. The true-tracking position $\theta_{T}$ and slope-corrected backtracking position are shown together for comparison. The backtracking correction $\theta_{c}$ is the angle between the backtracking and true-tracking rotations.

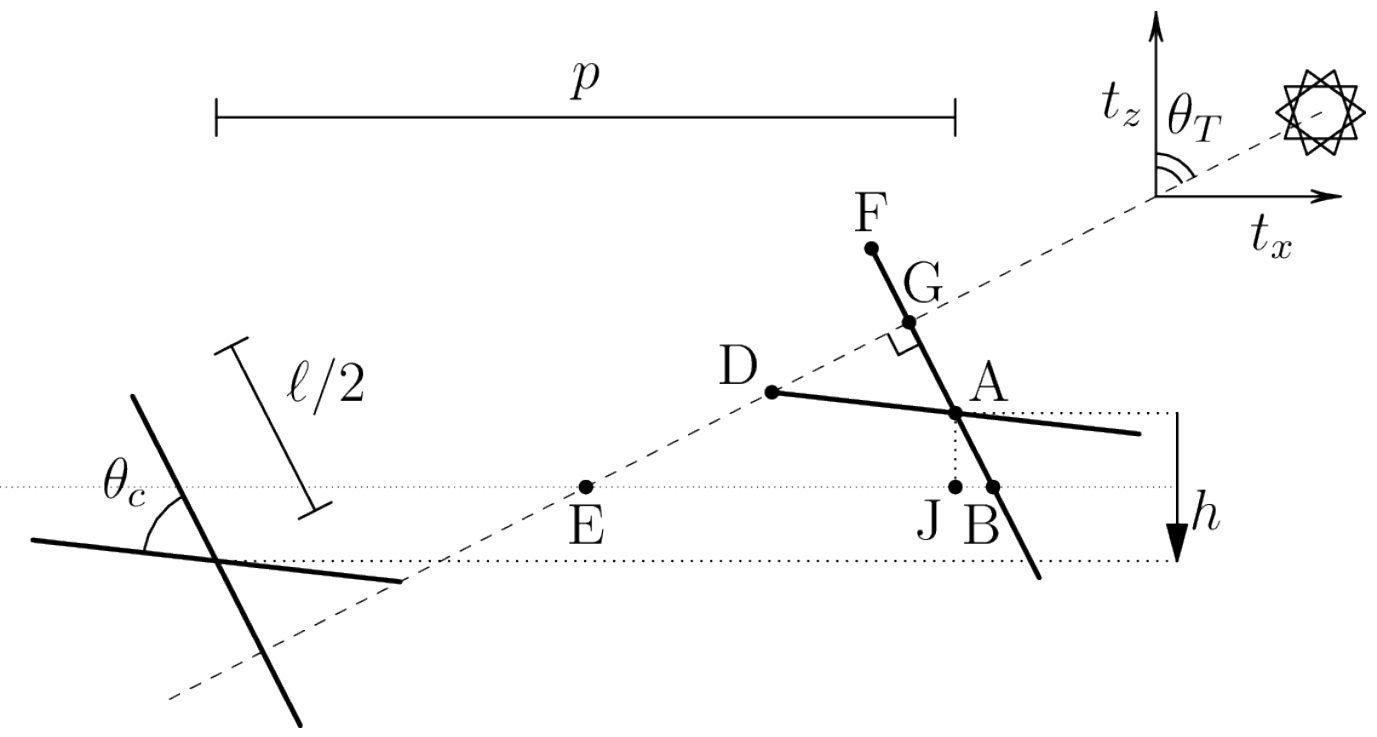

Figure 6. Cross-section of adjacent single-axis tracker rows with an offset $h$. The tracker axes point into the page and are not visible in this 2D diagram. The slope-aware backtracking position is shown in position $D$, with the true-tracking position as $F$ for comparison. Point $E$ shows the midpoint of the line segment (not shown) connecting the tracker axes, i.e. the segment between point $A$ and the corresponding point on the left tracker.

Note that, unlike the row pitch $p$, the row offset $h$ is a signed quantity depending on the direction of the cross-axis slope as determined by the right-hand rule around $\overrightarrow{t_{y}}$. Because $\overrightarrow{t_{y}}$ points into the page, a positive $\beta_{c}$ appears as a clockwise rotation from the reader's perspective. The slope in Figure 6 is rotated counter-clockwise from the reader's perspective, so $\beta_{c}$ is negative, and therefore we define $h$ as negative as well so it points down. For example, consider an array built on a west-facing slope $\left(\gamma_{g}=270\right)$ with tracker axis azimuth pointing south $\left(\gamma_{a}=180\right)$. The plane containing the tracker axes is tilted by a positive angle $\beta_{c}=\tan ^{-1} h / p$ compared to the horizontal plane by the right hand rule, and therefore $h$ would be positive (pointing up). If the slope were east-facing (again with tracker axis azimuth pointing south), the tracker axes plane 
would be tilted negative, and $h$ would also be negative (pointing down). Following these rules, Figure 6 shows a negative $h: \overrightarrow{t_{y}}$ points into the page, so the right hand rule determines that the axes plane is rotated negatively.

Similarly, many of the segment lengths in the following equations can be negative; we use $\overline{A B}$ to refer to this length and $|A B|$ in the cases where the length is always positive.

Finally, it is important to realize that $h$ and $\beta_{c}$ are defined by the offset along the $\overrightarrow{t_{z}}$ axis (i.e., in the tracker reference frame), making it necessary to consider the axis tilt $\beta_{a}$ when calculating them. Section 5 goes into more detail about calculating the cross-axis slope of tilted arrays.

The backtracking correction angle $\theta_{c}=\angle D A G$ is found by considering the right triangle AGD:

$$
\cos \theta_{c}=\frac{\overline{A G}}{\overline{A D}}
$$

Realizing $|A D|=\ell / 2$, we next find $\overline{A G}$ through triangle $E B G$. Because $\angle E B G=\theta_{T}$ :

$$
\cos \theta_{T}=\frac{\overline{B G}}{\overline{B E}}=\frac{\overline{B A}+\overline{A G}}{\overline{B J}+\overline{J E}}
$$

we have:

$$
\overline{A G}=(\overline{B J}+\overline{J E}) \cos \theta_{T}-\overline{B A}
$$

Because point $E$ is defined to be the midpoint between the tracker axes, by symmetry we have $|J E|=p / 2$ and $\overline{J A}=-h / 2 . \overline{B J}$ and $\overline{B A}$ are found by considering triangle $A J B$ and recognizing that $\angle A B J=\theta_{T}$ :

Then we have

$$
\overline{B J}=-\frac{h / 2}{\tan \theta_{T}} \quad \text { and } \quad \overline{B A}=-\frac{h / 2}{\sin \theta_{T}}
$$

$$
\begin{aligned}
\overline{A G} & =(|J E|+\overline{B J}) \cos \theta_{T}-\overline{B A} \\
& =\left(\frac{\mathrm{p}}{2}-\frac{\mathrm{h} / 2}{\tan \theta_{T}}\right) \cos \theta_{T}+\frac{h / 2}{\sin \theta_{T}} \\
& =\frac{\mathrm{p}}{2}\left[\left(1-\frac{h / p}{\tan \theta_{T}}\right) \cos \theta_{T}+\frac{h / p}{\sin \theta_{T}}\right] \\
& =\frac{p}{2}\left[\cos \theta_{T}-\frac{h}{p}\left(\frac{\cos \theta_{T}}{\tan \theta_{T}}-\frac{1}{\sin \theta_{T}}\right)\right] \\
& =\frac{\mathrm{p}}{2}\left[\cos \theta_{T}-\frac{h}{p}\left(\frac{\cos ^{2} \theta_{T}-1}{\sin \theta_{T}}\right)\right]
\end{aligned}
$$




$$
=\frac{\mathrm{p}}{2}\left[\cos \theta_{T}+\frac{h}{p} \sin \theta_{T}\right]
$$

Using the definitions $G C R=\ell / p$ and $\tan \beta_{c}=h / p$, we can now solve for the backtracking correction angle:

$$
\cos \theta_{c}=\frac{\cos \theta_{T}+\tan \beta_{c} \sin \theta_{T}}{G C R}
$$

or using the cosine angle addition identity:

$$
\cos \theta_{c}=\frac{\cos \left(\theta_{T}-\beta_{c}\right)}{G C R \cos \beta_{c}}
$$

Note that GCR is defined here as the ratio of collector width $\ell$ to horizontal separation $p$. This means that GCR remains a measure of array ground coverage when viewed from overhead; it ignores the vertical dimension. Also note that when the $\tan \beta_{c}$ is zero, the backtracking correction reverts to the original formula derived on horizontal ground.

However, because cosine is periodic and even, Equation 11 is satisfied by four distinct tracker rotations. For a given tracker rotation that satisfies Equation 11, its $180^{\circ}$ opposite will satisfy it as well, despite facing away from the sun. The second pair is generated by mirroring the first pair across the true-tracking plane (i.e.: by rotating forward by the correction angle instead of backward). The mirrored pairs have equivalent shading and direct irradiance capture characteristics, as shown in Figure 7:

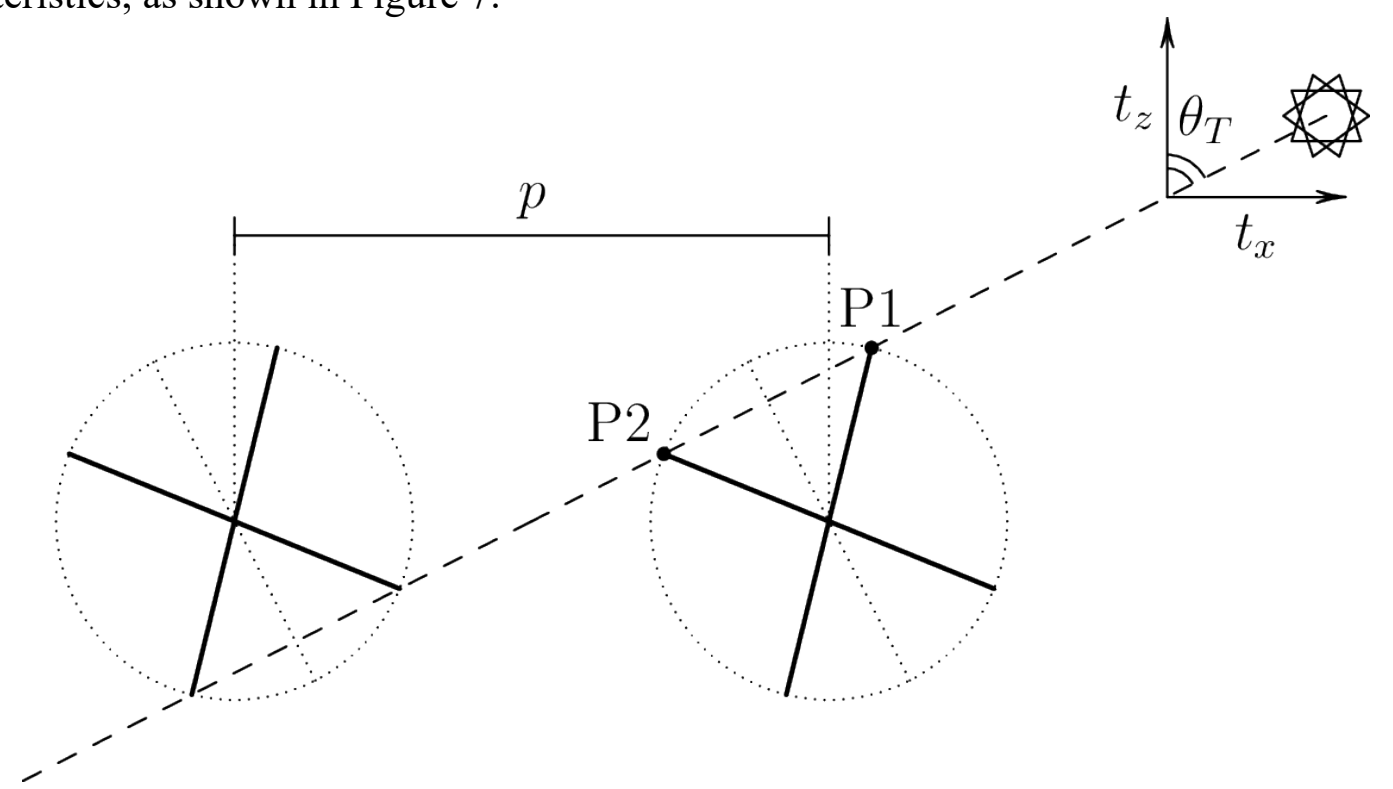

Figure 7. Cross-section of two adjacent tracker rows. The tracker axes point into the page and are not visible in this 2D diagram. The two optimal positions P1 and P2 eliminate row to row shading.

$P 1$ and $P 2$ expose the same cross section to beam irradiance and are symmetrical around the true-tracking position, shown as a dotted line perpendicular to the solar vector. 
First, we will eliminate rotations that face away from the sun. These rotations come about in cases where the plane containing the tracker axes (the "system plane") is not horizontal and the sun is above the horizon but below the system plane. In these cases $\cos \left(\theta_{T}-\beta_{c}\right)<0$, implying $\left|\theta_{c}\right|>90$ in Equation 11, so the tracker will have crossed the system plane and presented the row's rear side to the sun. However, because cosine is periodic, we are free to take the absolute value of the right-hand side (which is equivalent to adding $180^{\circ}$ to $\theta_{C}$ ) while still satisfying the equation. The effect is to keep $\left|\theta_{c}\right| \leq 90$ so that the front surface of the row will always see the sun (or, at worst, edge-on to it):

$$
\cos \theta_{c}=\frac{\left|\cos \left(\theta_{T}-\beta_{c}\right)\right|}{G C R \cos \beta_{c}}
$$

The remaining two correction angles are given by:

$$
\theta_{c}= \pm \cos ^{-1}\left(\frac{\left|\cos \left(\theta_{T}-\beta_{c}\right)\right|}{G C R \cos \beta_{c}}\right)
$$

One of the two positions is the standard backtracking position, but (by virtue of the $\theta_{c}$ derivation) the other is equal in shading avoidance and direct irradiance capture. Because it involves tracking forward beyond the true-tracking angle rather than behind it, it could be called "over-tracking". Whereas backtracking tends to orient rows flatter and pointing up, overtracking tends to orient them steeper and, at the edges of the day, upside down.

Because of the mechanical complications of such extreme rotations and the supposition that more diffuse irradiance is available downwelling from above than upwelling from below, backtracking will be preferred for PV systems. For the backtracking correction angle to tend to cancel the true-tracking angle, the sign of the tracking adjustment must be opposite that of the true-tracking angle:

$$
\theta_{c}=-\operatorname{sign}\left(\theta_{T}\right) \cos ^{-1}\left(\frac{\left|\cos \left(\theta_{T}-\beta_{c}\right)\right|}{G C R \cos \beta_{c}}\right) .
$$

The final note is that in the middle of the day, the argument to the arccosine will be out of range because there is no row-to-row shading to avoid. When the backtracking criterion given by

$$
\left|\frac{\cos \left(\theta_{T}-\beta_{c}\right)}{G C R \cos \beta_{c}}\right|<1
$$

is not satisfied, $\theta_{c}$ is set to zero.

The overall rotation when backtracking $\theta_{B}$ is then given by the sum of the true-tracking angle $\theta_{T}$ and the backtracking correction angle $\theta_{C}$ :

$$
\theta_{B}=\theta_{T}+\theta_{c}
$$




\section{Axis Tilt and Cross Slope}

The axis tilt $\beta_{a}$ and cross slope angle $\beta_{c}$ are dependent on the grade slope angle $\beta_{g}$ and the azimuth difference between tracker axis and grade $\Delta \gamma=\gamma_{a}-\gamma_{g}$. In Figure 8, segment $A B$ is a tracker axis. Points $A, B, C$ are coplanar with the grade and points $B, C, D$ define a horizontal plane parallel to the x-y plane, with points $C$ and $D$ being directly downslope of and below $A$, respectively. Points $A, C, D$ are coplanar with the vertical x-z plane. Vector $\vec{N}$ is the unit normal to the slope plane while unit vector $\vec{v}$ is inside the slope plane and perpendicular to both $A B$ and $\vec{N}$. Note that because the axis tilt and cross slope are invariant to azimuthal rotations, we use a rotated version of the global coordinate system here for convenience.

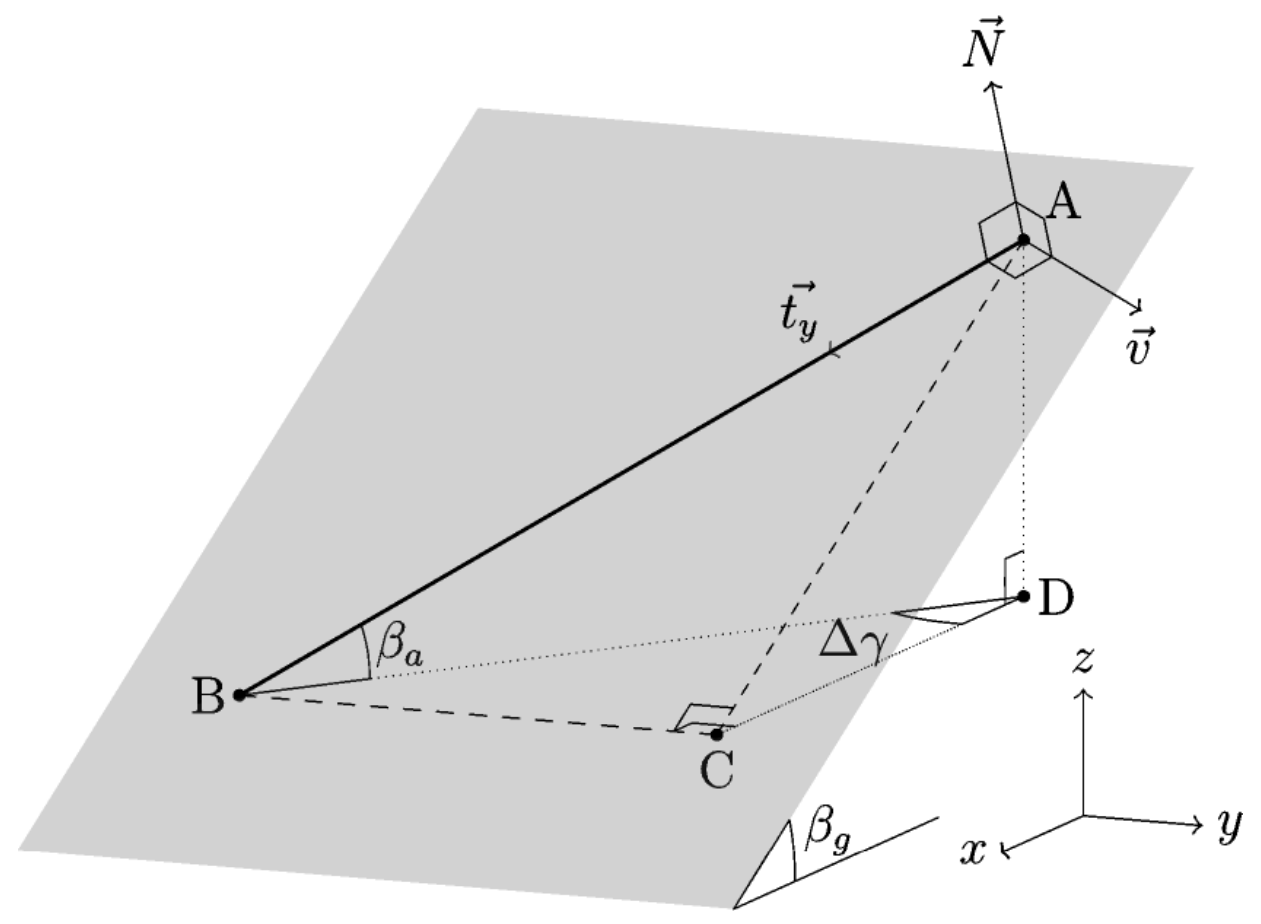

Figure 8. Geometry of a single-axis tracking axis $A B$ on a slope which is non-parallel to that axis. The vector $\vec{v}$ is perpendicular to $A B$ and parallel to the slope plane, representing the cross-axis slope vector. Note that the $(x, y, z)$ axes here are arbitrarily chosen to align with the slope.

Defining segment $A B$ to have length 1 , the leg lengths of the right triangle $A D C$ are:

$$
|A D|=\sin \beta_{a} \quad \text { and } \quad|\mathrm{CD}|=\cos \beta_{a} \cos \Delta \gamma
$$

Relating the grade slope angle $\beta_{g}=\angle A C D$ to these side lengths gives:

$$
\tan \beta_{g}=\frac{|A D|}{|C D|}=\frac{\tan \beta_{a}}{\cos \Delta \gamma}
$$

which can be rearranged to define $\beta_{a}$ :

$$
\beta_{a}=\tan ^{-1}\left(\tan \beta_{g} \cos \Delta \gamma\right)
$$


The cross-axis slope angle $\beta_{c}$ is found by calculating the cross product

$$
\vec{v}=\vec{N} \times \overrightarrow{t_{y}}
$$

where:

$$
\overrightarrow{t_{y}}=\left(\begin{array}{c}
\cos \beta_{a} \cos \Delta \gamma \\
-\cos \beta_{a} \sin \Delta \gamma \\
-\sin \beta_{a}
\end{array}\right) \quad \text { and } \quad \vec{N}=\left(\begin{array}{c}
\sin \beta_{g} \\
0 \\
\cos \beta_{g}
\end{array}\right)
$$

We choose to define $\vec{v}=\vec{N} \times \overrightarrow{t_{y}}$ over the alternative $\vec{v}=\overrightarrow{t_{y}} \times \vec{N}$ so that $\beta_{c}$ is a right-handed angle around $\overrightarrow{t_{y}}$. Taking Figure 8 as an example, using the right-hand rule and assuming the axis azimuth is along the downhill direction of the axis, the cross slope angle must be negative.

For ease of implementation, Equation 20 expands to:

$$
\vec{v}=\left[\begin{array}{l}
v_{x} \\
v_{y} \\
v_{z}
\end{array}\right]=\left[\begin{array}{c}
\sin \Delta \gamma \cos \beta_{a} \cos \beta_{g} \\
\sin \beta_{a} \sin \beta_{g}+\cos \Delta \gamma \cos \beta_{a} \cos \beta_{g} \\
-\sin \Delta \gamma \sin \beta_{g} \cos \beta_{a}
\end{array}\right]
$$

Because the vector $\vec{v}$ points across the trackers in the direction of the neighboring tracker axis, it defines the cross slope with its vertical component. However, as noted in Section 4, the crossaxis slope angle $\beta_{c}$ must be calculated in the tracker reference frame to be consistent with Equation 14. Therefore, we rotate $\vec{v}$ to adjust for the effect of the tracker axis tilt (rotate by $-\beta_{a}$ around the global $y$ axis) and azimuth difference (rotate by $\Delta \gamma$ around the global $z$ axis):

$$
\begin{aligned}
\mathbf{R}_{\boldsymbol{y}}=\left[\begin{array}{ccc}
\cos \beta_{a} & 0 & -\sin \beta_{a} \\
0 & 1 & 0 \\
\sin \beta_{a} & 0 & \cos \beta_{a}
\end{array}\right] & \text { and } \quad \mathbf{R}_{\mathbf{z}}=\left[\begin{array}{ccc}
\cos \Delta \gamma & -\sin \Delta \gamma & 0 \\
\sin \Delta \gamma & \cos \Delta \gamma & 0 \\
0 & 0 & 1
\end{array}\right] \\
& \overrightarrow{v^{\prime}}=\mathbf{R}_{\boldsymbol{y}} \mathbf{R}_{\mathbf{z}} \vec{v}
\end{aligned}
$$

Now that the cross-axis vector has been rotated into the tracker reference frame, the cross-axes slope is calculated with:

$$
\beta_{c}=\sin ^{-1} \frac{v_{z}^{\prime}}{\left|\overrightarrow{v^{\prime}}\right|}
$$

To simplify the calculation, we can exploit the fact that rotation matrices do not scale the rotated vector, so $|\vec{v}|=\left|\overrightarrow{v^{\prime}}\right|$, allowing us to skip the calculation of $v_{x}^{\prime}$ and $v_{y}^{\prime}$ :

$$
\beta_{c}=\sin ^{-1}\left(\frac{\left[v_{x} \cos \Delta \gamma-v_{y} \sin \Delta \gamma\right] \cdot \sin \beta_{a}+v_{z} \cos \beta_{a}}{|\vec{v}|}\right)
$$




\section{Shaded Fraction}

Again, extending the work provided by Lorenzo, Narvarte, and Muñoz (2011), we derive the shaded fraction of module area for trackers on a cross-axis slope at any rotation $\theta$. In Figure 9, the right tracker is shown raised above its horizontal-terrain analog.

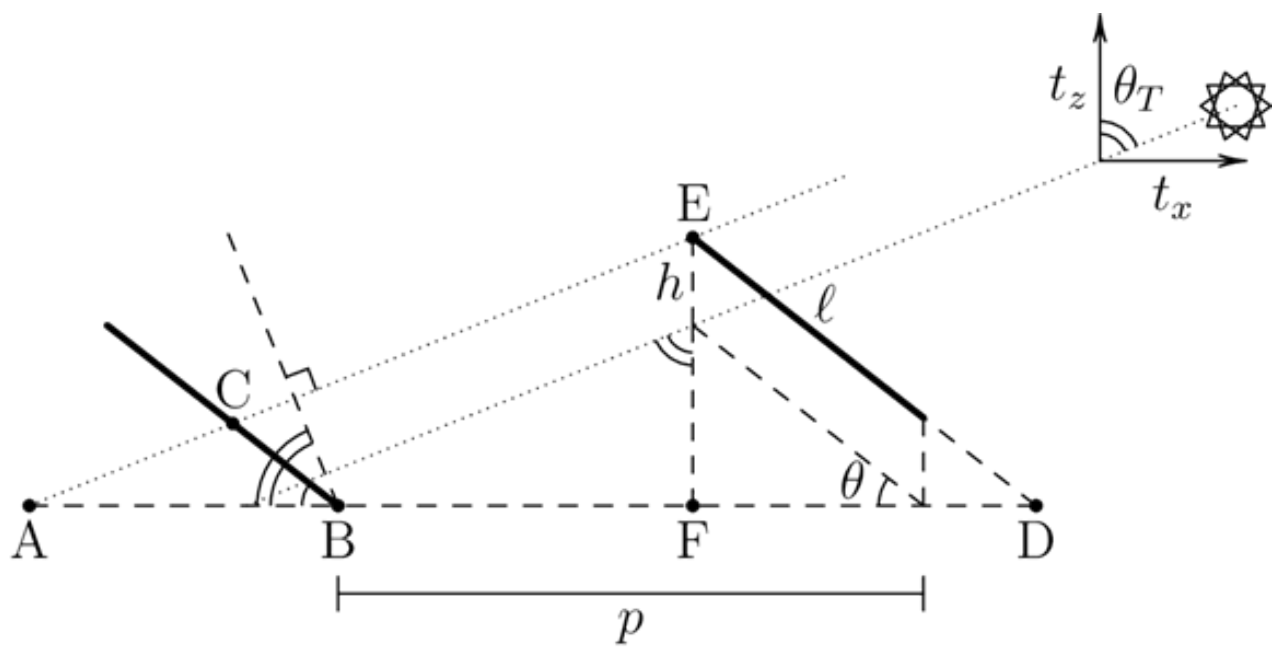

Figure 9. A cross-section of two adjacent tracker rows on a cross-axis slope. The tracker axes point into the page and are not visible in this 2D diagram. The tracker on the right is offset by $h$.

To calculate the shaded fraction $f_{s}=\overline{C B} / \ell$ of a tracker in some position $\theta=\angle A B C$ with zoffset $h=p \tan \beta_{c}$ when the true-tracking angle is $\theta_{T}$, realize that triangles $A B C$ and $A D E$ are similar and that the ratios of their corresponding side lengths are equal:

$$
\frac{\overline{C B}}{\overline{A B}}=\frac{\overline{\mathrm{ED}}}{\overline{\mathrm{AD}}}
$$

giving:

$$
f_{s}=\frac{1}{\ell} \frac{\overline{E D} \overline{A B}}{\overline{A D}}
$$

Using the side lengths given by:

$$
\begin{gathered}
\overline{E D}=\ell-h / \sin \theta \\
\overline{A D}=\overline{A F}+\overline{F D}=(\ell \sin \theta-h) \tan \theta_{T}+(\ell \cos \theta+\mathrm{h} \cot \theta) \\
\overline{A B}=\overline{A F}-\overline{B F}=(\ell \sin \theta-h) \tan \theta_{T}-(\mathrm{p}-\ell \cos \theta),
\end{gathered}
$$

and simplifying, the shaded fraction is given by:

$$
f_{s}=\max \left(0, \min \left(\frac{G C R \cos \theta+\left(G C R \sin \theta-\tan \beta_{C}\right) \tan \theta_{T}-1}{G C R\left(\sin \theta \tan \theta_{T}+\cos \theta\right)}, 1\right)\right)
$$

Note that $f_{s}$ must be clamped to the range $[0,1]$ to make physical sense as the shaded fraction. 


\section{Implementation Procedure}

Here we outline the process of calculating a tracker's true- and backtracking rotation angles.

1) Using the array's location and the datetime of interest, calculate the solar azimuth $\gamma_{s}$ and elevation $\beta_{s}$ with ephemeris tables or a solar position algorithm like that described in Reda and Andreas (2014).

2) If the array is mounted on a cross-axis slope, calculate the slope-adjusted axis tilt $\beta_{a}$ and cross-axis slope angle $\beta_{c}$ using the slope tilt $\beta_{g}$, the azimuth difference $\Delta \gamma=\gamma_{a}-\gamma_{g}$, and equations 19, 22, and 26:

$$
\begin{gathered}
\beta_{a}=\tan ^{-1}\left(\tan \beta_{g} \cos \Delta \gamma\right) \\
\vec{v}=\left[\begin{array}{l}
v_{x} \\
v_{y} \\
v_{z}
\end{array}\right]=\left[\begin{array}{c}
\sin \Delta \gamma \cos \beta_{a} \cos \beta_{g} \\
\sin \beta_{a} \sin \beta_{g}+\cos \Delta \gamma \cos \beta_{a} \cos \beta_{g} \\
-\sin \Delta \gamma \sin \beta_{g} \cos \beta_{a}
\end{array}\right] \\
\beta_{c}=\sin ^{-1}\left(\frac{\left[v_{x} \cos \Delta \gamma-v_{y} \sin \Delta \gamma\right] \cdot \sin \beta_{a}+v_{z} \cos \beta_{a}}{|\vec{v}|}\right)
\end{gathered}
$$

3) Calculate the solar projection and true-tracking angle using equations 1,4 , and 5 :

$$
\begin{gathered}
{\left[\begin{array}{l}
s_{x} \\
s_{y} \\
s_{z}
\end{array}\right]=\left[\begin{array}{c}
\cos \beta_{s} \sin \gamma_{s} \\
\cos \beta_{s} \cos \gamma_{s} \\
\sin \beta_{s}
\end{array}\right] .} \\
{\left[\begin{array}{c}
s_{x}^{\prime} \\
s_{y}^{\prime} \\
s_{z}^{\prime}
\end{array}\right]=\left[\begin{array}{c}
s_{x} \cos \gamma_{a}-s_{y} \sin \gamma_{a} \\
s_{x} \sin \gamma_{a} \cos \beta_{a}+s_{y} \cos \beta_{a} \cos \gamma_{a}-s_{z} \sin \beta_{a} \\
s_{x} \sin \gamma_{a} \sin \beta_{a}+s_{y} \sin \beta_{a} \cos \gamma_{a}+s_{z} \cos \beta_{a}
\end{array}\right]} \\
\theta_{T}=\operatorname{atan} 2\left(s_{x}^{\prime}, s_{z}^{\prime}\right)
\end{gathered}
$$

4) Calculate the preferred backtracking correction angle using equation 14:

$$
\theta_{c}=-\operatorname{sign}\left(\theta_{T}\right) \cos ^{-1}\left(\frac{\left|\cos \left(\theta_{T}-\beta_{c}\right)\right|}{G C R \cos \beta_{c}}\right) \text {. }
$$

5) Determine if backtracking is required by checking the condition in equation 15:

$$
\left|\frac{\cos \left(\theta_{T}-\beta_{c}\right)}{G C R \cos \beta_{c}}\right|<1
$$

6) Calculate the overall tracker rotation using equation 16:

$$
\theta_{B}=\theta_{T}+\theta_{c}
$$


Figure 10 shows several example tracker rotation curves calculated with the above procedure for the date 2019-01-01 at coordinates $(40.0,-80.0)$ with $G C R=0.5$ and $\beta_{g}=10^{\circ}$ at various tracker axis and slope azimuth.



Figure 10: Example true-tracking and backtracking curves for various array orientations.

Table 1: Validation data set for example in Figure 10 with $\gamma_{a}=195^{\circ}$ and $\gamma_{g}=180^{\circ}$.

\begin{tabular}{|l|l|l|l|l|}
\hline Time (UTC-05:00) & Apparent Elevation & Solar Azimuth & True-Tracking & Backtracking \\
\hline 8 AM & 2.404287 & 122.791770 & -84.440 & -10.899 \\
\hline 9 AM & 11.263058 & 133.288729 & -72.604 & -25.747 \\
\hline 10 AM & 18.733558 & 145.285552 & -59.861 & -59.861 \\
\hline 11 AM & 24.109076 & 158.939435 & -45.578 & -45.578 \\
\hline 12 PM & 26.810735 & 173.931802 & -28.764 & -28.764 \\
\hline 1 PM & 26.482495 & 189.371536 & -8.475 & -8.475 \\
\hline 2 PM & 23.170447 & 204.136810 & 15.120 & 15.120 \\
\hline 3 PM & 17.296785 & 217.446538 & 39.562 & 39.562 \\
\hline 4 PM & 9.461862 & 229.102218 & 61.587 & 32.339 \\
\hline 5 PM & 0.524817 & 239.330401 & 79.530 & 5.490 \\
\hline
\end{tabular}

axis tilt $\beta_{a}=9.666^{\circ}$

cross-axis slope angle $\beta_{c}=-2.576^{\circ}$ 


\section{References}

E. Lorenzo, L Narvarte, and J Muñoz. 2011. "Tracking and back-tracking." Progress in Photovoltaics: Research and Applications 19: 747-753. https://doi.org/10.1002/pip.1085

W. Marion and A. Dobos. 2013. "Rotation Angle for the Optimum Tracking of One-Axis Trackers." NREL Technical Report NREL/TP-6A20-58891. https://doi.org/10.2172/1089596

D. Schneider. 2012. "Control Algorithms for Large-scale Single-axis Photovoltaic Trackers." Acta Polytechnica, vol. 52, no. 5.

B. Nascimento, D. Albuquerque, M. Lima, P. Sousa. 2015. "Backtracking Algorithm for SingleAxis Solar Trackers installed in a sloped field." Int. Journal of Engineering Research and Applications, vol. 5, no. 12.4, pp. 100-103.

I. Reda and A. Andreas. 2004. "Solar position algorithm for solar radiation applications." Solar Energy, vol. 76, no. 5, pp. 577-589. https://doi.org/10.1016/j.solener.2003.12.003 